\title{
Photoacoustic microscopy of single cells employing an intensity-modulated diode laser
}

Langer, Gregor; Buchegger, Bianca; Jacak, Jaroslaw; Dasa, Manoj Kumar; Klar, Thomas A.; Berer, Thomas

Published in:

Proceedings of SPIE

Link to article, DOI:

$10.1117 / 12.2289978$

Publication date:

2018

Document Version

Publisher's PDF, also known as Version of record

Link back to DTU Orbit

Citation (APA):

Langer, G., Buchegger, B., Jacak, J., Dasa, M. K., Klar, T. A., \& Berer, T. (2018). Photoacoustic microscopy of single cells employing an intensity-modulated diode laser. In Proceedings of SPIE (Vol. 10494). [104942L] SPIE - International Society for Optical Engineering. Proceedings of SPIE, the International Society for Optical Engineering Vol. 104942 https://doi.org/10.1117/12.2289978

\section{General rights}

Copyright and moral rights for the publications made accessible in the public portal are retained by the authors and/or other copyright owners and it is a condition of accessing publications that users recognise and abide by the legal requirements associated with these rights.

- Users may download and print one copy of any publication from the public portal for the purpose of private study or research.

- You may not further distribute the material or use it for any profit-making activity or commercial gain

- You may freely distribute the URL identifying the publication in the public portal 


\section{Photoacoustic microscopy of single cells employing an intensity- modulated diode laser}

Gregor Langer, Bianca Buchegger, Jaroslaw Jacak, Manoj Kumar Dasa, Thomas A. Klar, et al.

Gregor Langer, Bianca Buchegger, Jaroslaw Jacak, Manoj Kumar Dasa, Thomas A. Klar, Thomas Berer, "Photoacoustic microscopy of single cells employing an intensity-modulated diode laser," Proc. SPIE 10494, Photons Plus Ultrasound: Imaging and Sensing 2018, 104942L (19 February 2018); doi: $10.1117 / 12.2289978$

SPIE. Event: SPIE BiOS, 2018, San Francisco, California, United States 


\title{
Photoacoustic microscopy of single cells employing an intensity- modulated diode laser
}

\author{
Gregor Langer*a , Bianca Buchegger ${ }^{\mathrm{b}}$, Jaroslaw Jacak ${ }^{\mathrm{b}, \mathrm{c}}$, Manoj Kumar Dasa ${ }^{\mathrm{d}}$, Thomas A. Klar ${ }^{\mathrm{b}}$, \\ Thomas Berer ${ }^{\mathrm{a}}$ \\ aResearch Center for Non-Destructive Testing GmbH, Altenberger Straße 69, 4040 Linz, Austria; \\ bInstitute for Applied Physics, Johannes Kepler University Linz, Altenberger Straße 69, 4040 Linz, \\ Austria; 'University of Applied Sciences Upper Austria, Applied Health \& Social Sciences, \\ Garnisonstraße 21, 4020 Linz; ${ }^{\mathrm{d} D e p a r t m e n t ~ o f ~ P h o t o n i c s ~ E n g i n e e r i n g, ~ T e c h n i c a l ~ U n i v e r s i t y ~ o f ~}$ \\ Denmark, 2800 Kgs. Lyngby, Denmark
}

\begin{abstract}
In this work, we employ frequency-domain photoacoustic microscopy to obtain photoacoustic images of labeled and unlabeled cells. The photoacoustic microscope is based on an intensity-modulated diode laser in combination with a focused piezo-composite transducer and allows imaging of labeled cells without severe photo-bleaching. We demonstrate that frequency-domain photoacoustic microscopy realized with a diode laser is capable of recording photoacoustic images of single cells with sub- $\mu \mathrm{m}$ resolution. As examples, we present images of undyed human red blood cells, stained human epithelial cells, and stained yeast cells.
\end{abstract}

Keywords: photoacoustic microscopy, frequency-domain, diode laser

\section{INTRODUCTION}

Photoacoustic microscopy is capable of providing label-free imaging by utilizing the endogenous contrast of chromophores to be imaged. Endogenous molecules absorb the light at the excitation wavelength, leading to a temperature increase which finally causes acoustic waves. In principle, any substance exhibits optical absorbance at a certain wavelength and can thus be imaged. Cell nuclei, for example, exhibit strong absorption at a light wavelength of $266 \mathrm{~nm}^{1}$. Hemoglobin, as another example, has a strong absorption in the spectral region around $410 \mathrm{~nm}$. Hence, using a suitable wavelength of the excitation light, label-free photoacoustic imaging could be realized for every molecule. In fluorescence microscopy, most samples require labelling with chromophores as most absorbing molecules do not exhibit fluorescence. In photoacoustic microscopy, staining may be necessary if the excitation light is not or only weakly absorbed by the investigated structures. Using specialized stains allows to tag proteins, lipids, DNA, etc., thereby enabling visualization of specific cellular components. However, most of the fluorescent dyes are toxic to the cell or at least influence its chemical behavior. This poses a problem for in vivo research. Therefore, there is an ongoing search for less toxic stains, e.g. quantum-dots or nanoparticles, which exhibit much less or no fluorescence signal, but could be used as photoacoustic labels. In this manuscript, we show images of unlabeled and labeled single cells. The unlabeled cells are red blood cells which do well absorb the used excitation light with a wavelength of $405 \mathrm{~nm}$. Yeast cells show only weak intrinsic absorption at $405 \mathrm{~nm}$ and were, therefore, stained with Rhodamine or Atto dyes in order to enable photoacoustic signal acquisition.

Photoacoustic micrographs of single cells are mainly recorded with photoacoustic microscopes realized in time-domain, i.e. with pulsed laser sources. In time-domain much larger photoacoustic pressures can be generated ${ }^{2}$. However, recently it was reported that frequency-domain PAM can achieve similar signal-to-noise ratios under the fulfillment of several requirements ${ }^{3}$. It is well known that hemoglobin provides strong optical absorption in the visible and gives an excellent photoacoustic contrast. Various groups have used this high endogenous contrast for demonstrating photoacoustic imaging of single red blood cells using time-domain $\mathrm{PAM}^{4-8}$. Frequency-domain PAM of red blood cells has been demonstrated by Winkler $e t a l .{ }^{9}$ and Langer $e t a l^{3}$.

Pulsed laser sources employed for PAM usually are rather expensive. In the present paper, we employ an intensity modulated diode laser. Although the generated photoacoustic pressure is many orders of magnitudes below that of pulsed lasers $^{2}$ the signal to noise ratio (SNR) is comparable, as we utilize the noise-rejection capabilities of lock-in techniques.

*gregor.langer@recendt.at; phone 00437322468 4656; www.recendt.at

Photons Plus Ultrasound: Imaging and Sensing 2018, edited by Alexander A. Oraevsky, Lihong V. Wang, Proc. of SPIE Vol. 10494, 104942L · @ 2018 SPIE · CCC code: 1605-7422/18/\$18 · doi: 10.1117/12.2289978 


\section{SET-UP}

In Fig. 1 a simplified sketch of the frequency-domain photoacoustic microscope is shown. A computer is connected via USB to a piezo-stage controller (PI, E-725) and receives measurement data from a lock-in-amplifier (LIA, Zurich Instruments, UHFLI). Via digital I/O lines, the piezo controller enables the laser output and synchronizes stage position with data acquisition. An arbitrary waveform generator (f-gen, Agilent, 33250A) is used to modulate the output intensity of the diode laser (Omicron, A350). The $405 \mathrm{~nm}$ light emitted from the diode laser is mode cleaned via a telescope and a $30 \mu \mathrm{m}$ high power pin hole (Edmund Optics, \#39-886) and subsequently focused onto the sample via an objective lens (Olympus, UPlanFl 20×, NA $=0.5$ or UPlanFl $40 \times, \mathrm{NA}=0.75$ ), providing sub- $\mu \mathrm{m}$ lateral resolution ${ }^{3}$. The sample is fixed on a microscope slide and placed onto a nanopositioning system (PIMars, P-563.3CD) which permits three-dimensional scanning. In Fig. 1, the piezo stage is symbolized by a three-dimensional coordinate system. Acoustic waves, which are generated in the sample are detected via a focusing hydrophone (Sonaxis, CMF 20MHz). The hydrophone's signal is amplified by an amplifier (Mini-Circuits, ZFL-500LN+) connected to the input of the LIA.

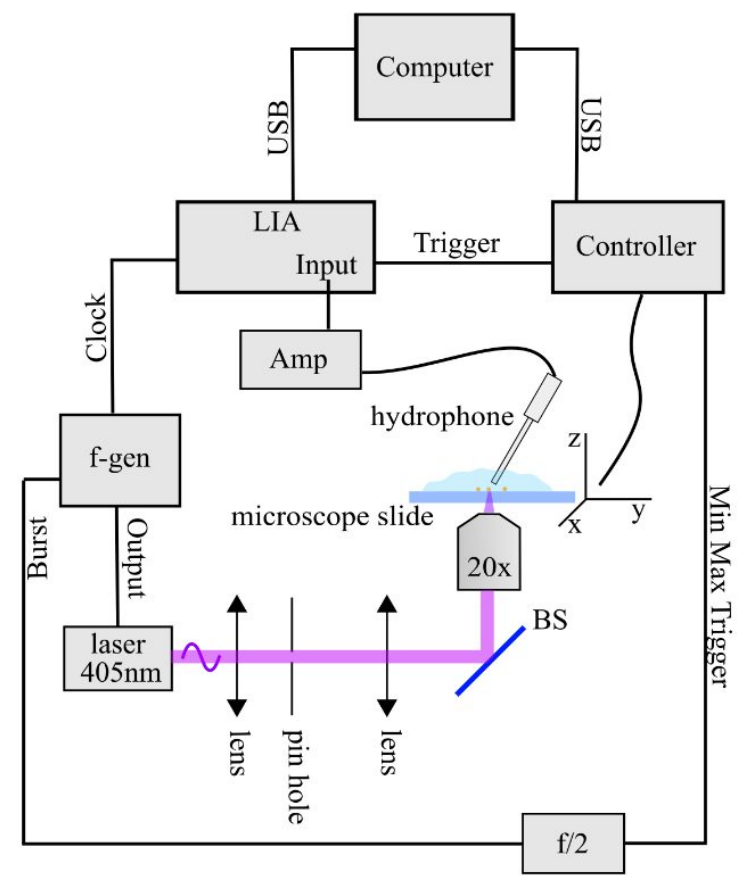

Figure 1. Schematic of the photoacoustic microscope. The output of a diode laser (laser $405 \mathrm{~nm}$ ) with a wavelength of $405 \mathrm{~nm}$ is modulated either sinusoidally or with a square-wave using an arbitrary waveform generator (f-gen). The sample is fixed on a microscope slide, positioned on a nanopositioning stage (x,y,z coordinate system), and scanned relative to the fixed laser beam. The laser beam is focused onto the sample with an objective lens (20x). Photoacoustic signals are recorded by the hydrophone, which are amplified (Amp) and fed into a lock-in amplifier (LIA). The LIA, as well as the stage controller (Controller), are controlled via a personal computer (Computer). At fixed stage distances, e.g. every $500 \mathrm{~nm}$, the controller sends trigger pulses, commanding the LIA to store the current input value at this position. The sample is exposed only either during the forward of the backward scan-direction of the fast axis. For the reverse direction, the laser output is disabled, using digital I/O lines provided by the stage controller.

Photoacoustic image data can be acquired during forward and backward motion of the piezoelectric stage. However, piezoelectric stages usually exhibit hysteresis regarding the position. This hysteresis increases with increasing scanning speed. If scanning is performed at high rates, the positions of the backward and forward scans can differ by several $\mu \mathrm{m}$. Additionally, the photoacoustic signal amplitude can differ between backward and forward scans causing stripe-shaped artifacts within the images (see e.g. Fig. 3). As the magnitude of these effects depend non-linearly on scan speed and signal amplitude respectively, these effects cannot be removed via simple image post-processing. In order to record distortion free images, only the forward or backward scan is considered.

In photoacoustic microscopy, high laser intensities are required in order to create photoacoustic signals. This can cause photo-bleaching or photo-damage. To reduce the incident light dose, we operate the arbitrary waveform generator in externally triggered burst-mode, whereby the external trigger is provided by the piezo-controller. The trigger signal is 
frequency divided by a custom built frequency divider. By this, the laser emits light only in forward scan direction while it is turned off during the backward scan. Fig. 2 and Figs. 4-6 were recorded accordingly.

\section{SIGNAL TO NOISE RATIO}

In the following, the signal to noise ratio (SNR) of an image is defined as the ratio between the mean value of the signal, $\mu_{\mathrm{sig}}$, and the standard deviation of the background $\sigma_{\mathrm{bg}}(\mathrm{RMS}-\mathrm{noise})$ :

$$
\mathrm{SNR}=\frac{\mu_{\text {sig }}}{\sigma_{\mathrm{bg}}}
$$

For the SNR determination, an area with high photoacoustic signal and a background area were chosen. From the highsignal area, the mean value was calculated. From the background area, the standard deviation was calculated. The SNR was then determined according to Eq. (1). The high-signal area and the background area were chosen arbitrarily. Therefore, evaluations using different sets of signal and background areas can result in different SNR values. This issue is considered by error estimation of the SNR. Within this manuscript, photoacoustic images with SNR values above 70 are shown. Anyhow, in order to extend this imaging method to new types of samples and new applications, we still search for possibilities to increase the measurement sensitivity even further. In the following, some measures of how to increase the sensitivity of frequency-domain photoacoustic microscopy are mentioned: The photoacoustic signal generation in frequency-domain does linearly increase with increasing excitation modulation frequency ${ }^{3}$, at least as long as the modulation frequency is below the inverse of the specimen's excited state lifetime ${ }^{10,11}$. In order to increase the SNR, in frequency-domain photoacoustic imaging, the highest possible modulation frequency should be used. However, the ultrasound attenuation of the coupling medium increases with increasing frequency ${ }^{12}$ and, thereby, determines the maximum possible ultrasound frequency. Consequently, to measure ultrasound frequencies above $100 \mathrm{MHz}$, the distance between excitation source and transducer has to be kept low, i.e. significantly below $10 \mathrm{~mm}^{5,11}$. Direct laser irradiation of the hydrophone may cause artifacts and poses a problem when using an ultrasound transducer too close to the excitation volume. Other measures to improve the SNR are the usage of square-wave intensity modulation instead of a pure sine modulation ${ }^{13,14}$ and, obviously, to increase the sensitivity of the ultrasound transducer. In this manuscript, we employed a focused piezo-composite transducer with a maximum sensitivity at $20 \mathrm{MHz}$ and a focal distance of approx. $10 \mathrm{~mm}$. Piezocomposite hydrophones with higher center frequencies, e.g. $>30 \mathrm{MHz}$, are expected to give even higher $\mathrm{SNR}^{11}$. For a more detailed discussion on the influence of ultrasonic attenuation on the optimum modulation frequency see, e.g., Langer et $a .^{11}$

\section{SINGLE CELL IMAGING}

By making use of the measures described in section 3, we managed to record photoacoustic micrographs of unlabeled and labeled single cells with reasonable signal-to-noise ratio. Note, that all presented images consist of the original measurement data. All images were created with MATLAB. With exception of Fig. 3, no image pre-processing, e.g. offset correction, or post-processing, e.g. brightness or contrast correction, was performed.

\subsection{Unlabeled red blood cells}

In Fig. 2 photoacoustic images of human red blood cells are shown. A drop of human blood was placed on a silanized microscope slide (Marienfeld Superior) and dispersed via a thin glass slide. The dry blood film was fixed with methanol in order to prevent red blood cells to dissolve in water, which is used for ultrasound coupling. The exposure time of a single pixel was $0.5 \mathrm{~ms}$. For the focusing, a $20 \times$ objective lens was used. The laser intensity was around $2.5 \mathrm{GW} / \mathrm{m}^{2}$. The signal to noise ratio of Fig. $2 \mathrm{a}$ is $47 \pm 7$. 

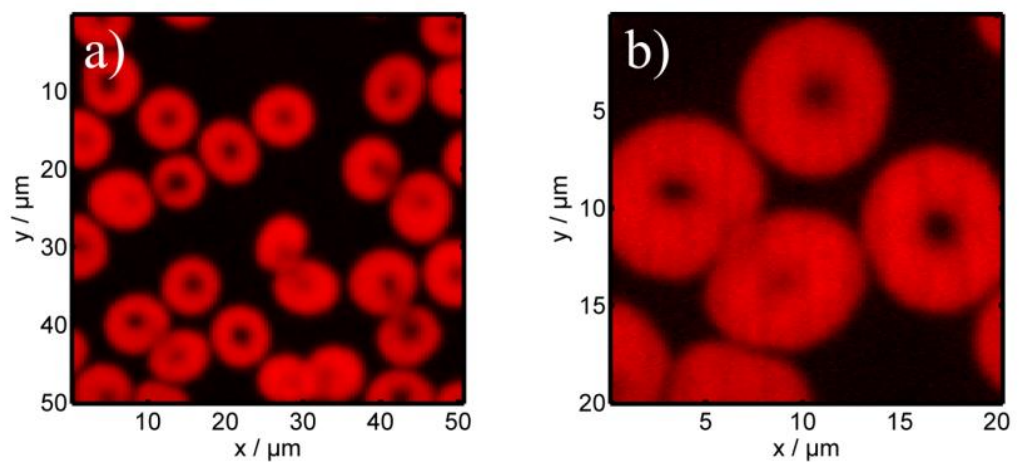

Figure 2. (a) Photoacoustic image of human red blood cells. (b) Detailed image of a region, recorded after image (a). In (b) the faint dark lines along the y-direction, i.e. the fast scanning direction, indicate photo-bleaching. On the images, no background noise is visible. Note that the images were neither pre- nor post-processed, nor was a color bar shift applied.

\subsection{Human epithelial cells labeled with Atto 425}

Saliva was placed on silanized microscope slides. After the water was evaporated, the cells were fixed with methanol for several minutes. Subsequently, the sample was washed in order to remove loose cells. The sample was then stained with NHS functionalized Atto 425 for several hours. Unbound stain was removed by rinsing the sample with ethanol. For image acquisition a 20x objective lens and a laser modulation frequency of $21.11 \mathrm{MHz}$ were used. The exposure time per pixel was $2 \mathrm{~ms}$. For this image, measurement data consisting of the backward and forward scans were used. Some strip artefact, caused due to hysteresis, can be recognized.

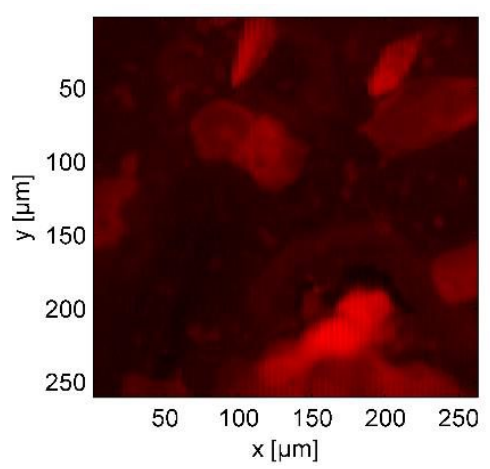

Figure 3. Photoacoustic image of epithelial cells from saliva stained with Atto 425. This image was brightness enhanced to enhance the visibility of structures with weaker signals.

Besides epithelial cells, other structures are also visible. These structures might be bacteria or cell organelles, e.g. cell nuclei.

\subsection{Yeast labeled with Atto390}

Yeast (Saccharomyces cerevisiae) was bought in a local supermarket and dissolved in water. Drops of yeast suspension were put onto silanized microscope slides. The water was allowed to evaporate at room temperature. Subsequently, the dried yeast was fixed with methanol for some minutes. A dye solution of NHS functionalized Atto 390 was applied at slightly elevated temperatures for several hours. Finally, the sample was rinsed with ethanol in order to remove the unbound stain. 


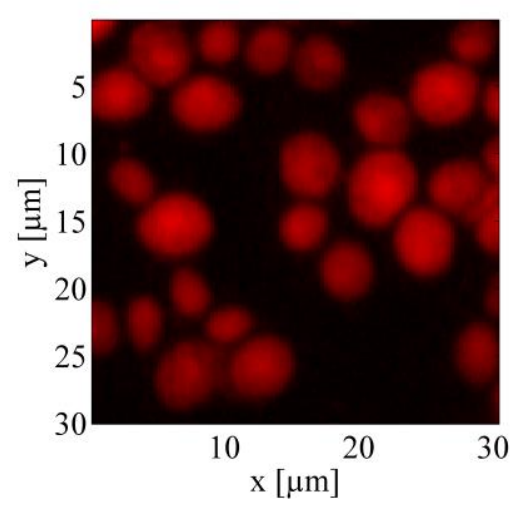

Figure 4. Photoacoustic image of yeast stained with Atto 390. The measurement data was neither pre- nor post-processed.

For image acquisition a $20 \times$ objective lens, a laser modulation of $20.1 \mathrm{MHz}$, and a laser intensity of around $2.5 \mathrm{GW} / \mathrm{m}^{2}$ were used. The exposure time per pixel was $0.36 \mathrm{~ms}$. The yeast cells possess diameters between 2 to $5 \mu \mathrm{m}$. In certain yeast cells, one can recognize lighter and darker areas.

\subsection{Yeast dyed with Rhodamine 6G}

Sample preparation was similar as described in section 4.2 (Yeast dyed with Atto 390), except that NHS functionalized Rhodamine 6G (ATTO-TEC, Rho6G-31) was used instead of Atto 390. In Fig. 5 the photoacoustic image of yeast stained with Rhodamine $6 \mathrm{G}$ is shown. For this image, a $40 \times$ objective lens was used. The exposure time per pixel was $0.375 \mathrm{~ms}$ and the modulation frequency was $20.1 \mathrm{MHz}$. No noise can be recognized in Fig. 5 with the bare eye, although the measurement data was neither pre- nor post-processed. However, darker and brighter areas within the cells can be identified. Comparison with a simultaneously acquired fluorescence image showed that the photoacoustic and fluorescence signals behave complementary, i.e. at positions where large fluorescence was observed the photoacoustic signals were lower and vice versa ${ }^{15}$. We attribute this effect to a spatially varying quantum yield of the stain, caused by influences of the local chemical environment.

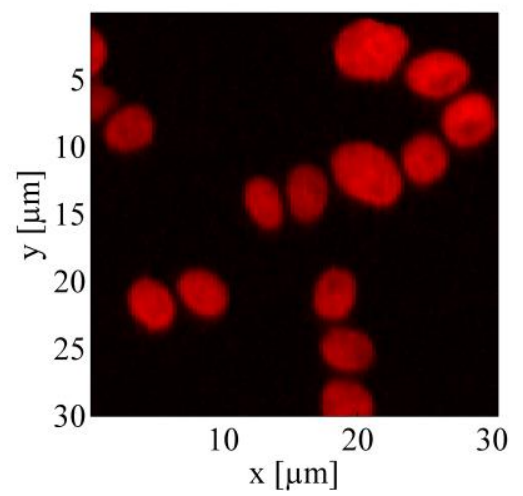

Figure 5. Photoacoustic image of yeast labeled with Rhodamine 6G. No background noise is visible to the bare eye. The measurement data was neither pre- nor post-processed.

\section{PHOTOBLEACHING}

Photoacoustic imaging demands much higher excitation intensities than fluorescence imaging. Therefore, photo-bleaching and photo-damage pose a challenge. In fluorescence imaging, photo-bleaching is mainly caused by the transformation of fluorescent molecules to non-fluorescent molecules, e.g. via thermally or photo-induced chemical reactions. In general, the amplitudes of the fluorescence and photoacoustic signals, $S_{\mathrm{FL}}$ and $S_{\mathrm{PA}}$, are proportional to the absorption coefficient, $\alpha$. The fluorescence signal is also proportional to the fluorescence quantum yield, $\eta$. For the photoacoustic signal, if neglecting photoacoustic signal generation via vibrational relaxation ${ }^{11}$, the signal is proportional to one minus the fluorescence quantum yield: 


$$
\begin{gathered}
S_{\mathrm{FL}} \propto \alpha \eta \\
S_{\mathrm{PA}} \propto \alpha(1-\eta)
\end{gathered}
$$

Due to the laser exposure the molecules may dissociate, undergo a transformation to the triplet state, or react with molecules which are present in the environment. This acts back onto the absorption coefficient and on the fluorescence quantum yield. Fluorescence dyes are designed such that the absorption cross section $(\alpha)$ and the fluorescence quantum yield $(\eta)$ are both high. A molecular transformation is, therefore, extremely likely to reduce at least one or both of these properties, which causes a deterioration of the fluorescence signal. Fluorescence photo-bleaching, however, may be accompanied with an increase of photoacoustic signal, e.g., if the decrease of the fluorescence quantum yield is the dominant bleaching process. If the absorption coefficient at the excitation wavelength increases, or if the fluorescence quantum yield decreases due to laser light exposure, the photoacoustic signal is likely to increase. If the absorption coefficient decreases or the fluorescence quantum yield increases, the photoacoustic signal does decrease.

Fig. 6 shows the photoacoustic image of the first (Fig. 6a) and sixth (Fig. 6b) exposure of yeast stained with Rhodamine 6G. The employed excitation laser intensity was approximately $2.5 \mathrm{GW} / \mathrm{m}^{2}$. The first exposure has an SNR of $73 \pm 3$. The sixth exposure (Fig. 6b) has a slightly lower SNR of $65 \pm 5$. Both images look similar and besides marginal photobleaching, no photo-damage was observed.
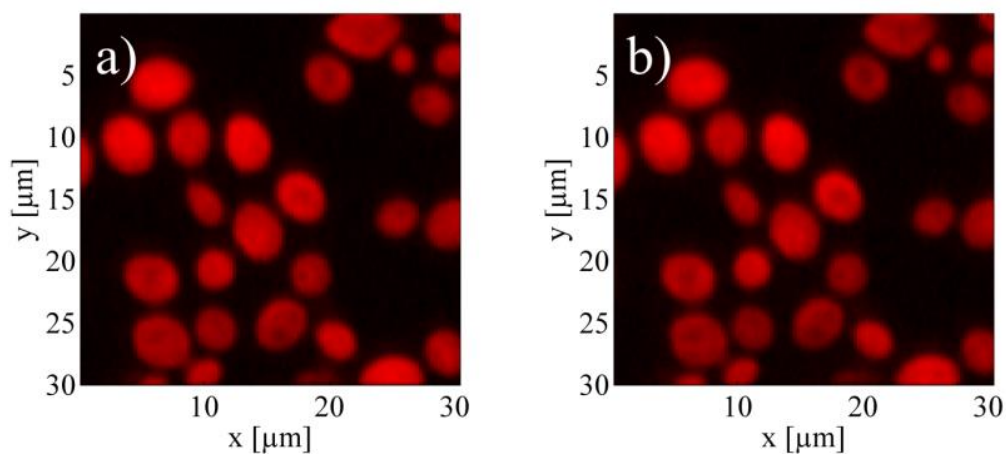

Figure 6. The photoacoustic signal of yeast stained with Rhodamine 6G. First exposure (a) and sixth exposure (b). For the image acquisition a $20 \times$ objective lens was used. The laser intensity was around $2.5 \mathrm{GW} / \mathrm{m}^{2}$.

\section{CONCLUSION}

Within this work, we demonstrated that it is possible to image single unlabeled and labeled cells, e.g. human red blood cells, stained epithelial cells, or stained yeast, by means of frequency-domain photoacoustic microscopy with reasonable SNR. As the excitation laser intensities required for signal generation are rather high, photo-bleaching could become an issue in photoacoustic microscopy. Nevertheless, it was demonstrated that recording several subsequent images of Rhodamine $6 \mathrm{G}$ stained yeast cells is feasible without significant photo-bleaching.

\section{ACKNOWLEDGEMENTS}

This work has been supported by the Austrian Science Fund (FWF), project number P27839-N36. This work has also been supported by the project "multimodal and in-situ characterization of inhomogeneous materials" (MiCi) by the federal government of Upper Austria and the European Regional Development Fund (EFRE) in the framework of the EU-program IWB2020. Additional support is acknowledged by the strategic economic- research program "Innovative Upper Austria 2020" of the province of Upper Austria. We thank Gerd Marienfeld from Marienfeld-Superior for providing us with silanized cover-slips. 


\section{REFERENCES}

[1] Yao, D.-K., Maslov, K., Shung, K.K., Zhou, Q., and Wang, L.V., "In vivo label-free photoacoustic microscopy of cell nuclei by excitation of DNA and RNA," Optics Letters 35(24), 4139-4141 (2010).

[2] Yao, J., and Wang, L.V., "Sensitivity of photoacoustic microscopy," Photoacoustics 2(2), 87-101 (2014).

[3] Langer, G., Buchegger, B., Jacak, J., Klar, T.A., and Berer, T., "Frequency domain photoacoustic and fluorescence microscopy," Biomedical Optics Express 7(7), 2692-2702 (2016).

[4] Rao, B., Maslov, K., Danielli, A., Chen, R., Shung, K.K., Zhou, Q., and Wang, L.V., "Real-time fourdimensional optical-resolution photoacoustic microscopy with Au-nanoparticle assisted sub-diffractionlimit resolution," Optics letters 36(7), 1137-1139 (2011).

[5] Strohm, E.M., Moore, M.J., and Kolios, M.C., "Single Cell Photoacoustic Microscopy: A Review," IEEE Journal of Selected Topics in Quantum Electronics 22(3), 137-151 (2016).

[6] Shelton, R.L., Mattison, S.P., and Applegate, B.E., "Volumetric imaging of erythrocytes using label-free multiphoton photoacoustic microscopy," Journal of Biophotonics 7(10), 834-840 (2014).

[7] Nedosekin, D.A., Galanzha, E.I., Dervishi, E., Biris, A.S., and Zharov, V.P., "Super-resolution nonlinear photothermal microscopy," Small 10(1), 135-142 (2014).

[8] He, G., Li, B., and Yang, S., "In vivo imaging of a single erythrocyte with high-resolution photoacoustic microscopy," Frontiers of Optoelectronics 8(2), 122-127 (2015).

[9] Winkler, A.M., Maslov, K., and Wang, L.V., "Noise-equivalent sensitivity of photoacoustics," Journal of Biomedical Optics 18(9), 097003 (2013).

[10] Langer, G., Buchegger, B., Jacak, J., Pfeffer, K., Klar, T.A., and Berer, T., "Multimodal fluorescence and photoacoustic microscopy in the frequency domain," Proc. SPIE 10057, 1005707 (2017)

[11] Langer, G., and Berer, T., "Influence of the excited state lifetimes on the photoacoustic signal," Proc. SPIE 10494, (2018).

[12] Bauer-Marschallinger, J., Berer, T., Grun, H., Roitner, H., Reitinger, B., and Burgholzer, P., "Broadband high-frequency measurement of ultrasonic attenuation of tissues and liquids," IEEE transactions on ultrasonics, ferroelectrics, and frequency control 59(12), 2631-2645 (2012).

[13] Langer, G., Langer, A., Buchegger, B., Jacak, J., Klar, T.A., and Berer, T., "Frequency domain optical resolution photoacoustic and fluorescence microscopy using a modulated laser diode," Proc. SPIE 10064, 1006426 (2017).

[13] Booth, M.J., and Wilson, T., "Low-cost, frequency-domain, fluorescence lifetime confocal microscopy," Journal of Microscopy 214(1), 36-42 (2004).

[15] Langer, G., Buchegger, B., Jacak, J., Pfeffer, K., Wohlfarth, S., Hannesschläger, G., Klar, T.A., and Berer, T., "Frequency-domain photoacoustic and fluorescence microscopy: Application on labeled and unlabeled cells," Proc. SPIE 10487, (2018). 